\title{
Zero-bias anomalies in electrochemically fabricated nanojunctions
}

\author{
L.H. Yu and D. Natelson \\ Department of Physics and Astronomy, Rice University, 6100 Main St., Houston, TX 77005
}

(Dated: October 30, 2018)

\begin{abstract}
A streamlined technique for the electrochemical fabrication of metal nanojunctions (MNJs) between lithographically defined electrodes is presented. The first low-temperature transport measurements in such structures reveal suppression of the conductance near zero-bias. The size of the zero-bias anomaly (ZBA) depends strongly on the fabrication electrochemistry and the dimensions of the resulting MNJ. We present evidence that the nonperturbative ZBA in atomic-scale junctions is due to a density of states suppression in the leads.
\end{abstract}

PACS numbers:

Metallic nanojunctions (MNJ) are unique tools for examining electronic transport, correlations, quantum coherence, and disorder at the atomic scale. These atomic size devices, with diameter and length much smaller than the electronic phase coherence length, are the subjects of current research. Extensive progress has been made in understanding clean MNJs fabricated and measured in ultrahigh vacuum (UHV) by the break junction method, using either scanning tunneling microscope 1] or microfabricated structures 2, 3].

Recently MNJs have also been prepared using electrochemistry and proposed for use in molecular electronic devices 4, 5, 6, 7, 8]. However, MNJs made in solution may be "dirty" due to grain boundaries and incorporation or surface adsorption of ionic impurities. While junctions made electrochemically exhibit conductance quantization and Ohmic behavior at room temperature 4, 5], low-temperature transport properties of these systems have yet to be examined.

In this letter we report a nanojunction fabrication method that synthesizes elements from various electrochemical approaches, and report low-temperature nanojunction conductance measurements. We find suppressions of the dc conductance near zero bias at low temperatures. In $\mathrm{Au}$ junctions made using an alkaline buffer solution 4$]$ and having conductance $G(300 \mathrm{~K})<\sim$ $2 e^{2} / h \equiv G_{0}$, this suppression approaches $100 \%$. Much smaller zero-bias anomalies (ZBAs) occur in Au junctions made with an $\mathrm{HCl}$ solution [7]. We show that the large ZBAs are caused by nonperturbative corrections to the local density of states of the leads.

The nanojunction fabrication process begins with gold source and drain electrodes defined by electron-beam lithography (EBL) on $200 \mathrm{~nm}$ of $\mathrm{SiO}_{2}$ on $p+$ silicon substrates. We define electrodes with tip widths $\sim 200 \mathrm{~nm}$ separated by $\sim 10 \mathrm{~nm}$. In an e-beam evaporator with base pressure of $10^{-7} \mathrm{mB}$, we then deposit $2.5 \mathrm{~nm}$ of titanium $(0.1 \mathrm{~nm} / \mathrm{s}), 25 \mathrm{~nm}$ of gold $(0.3 \mathrm{~nm} / \mathrm{s})$, and $20 \mathrm{~nm}$ of $\mathrm{Al}_{2} \mathrm{O}_{3}(0.3 \mathrm{~nm} / \mathrm{s})$, and perform liftoff. The electrodes have $R / \square \sim 1 \Omega$. A representative image of an electrode pair is in Fig. 1 An additional $0.1 \mathrm{~mm}^{2}$ pad $20 \mu \mathrm{m}$ from the tips of the electrodes is also evaporated to serve as an auxiliary electrode 6 .

Starting from these electrodes, electrochemistry is

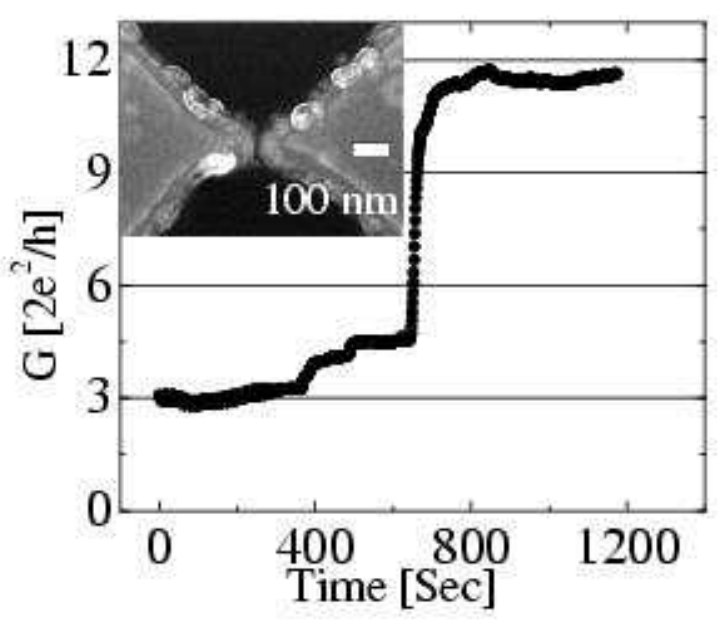

FIG. 1: Steps in junction conductance as atomic configurations shift during growth. Inset: micrograph of alumina-coated starting electrodes.

performed to produce a nanoscale junction. The low-temperature junction conductance properties are strongly influenced by the electrochemical protocol. Except where noted, the devices described in this letter are made using the following solution-based technique 4] (labeled "M"): The electrodes are covered with an aqueous solution of $0.01 \mathrm{M} \mathrm{KAu}(\mathrm{CN})_{2}$ in a buffer of $1 \mathrm{M} \mathrm{KHCO}_{3}$ and $0.2 \mathrm{M} \mathrm{KOH}(\mathrm{pH} \approx 10)$. Deposition current (up to $4 \mu \mathrm{A}$ ) is sourced via the auxiliary electrode, while maintaining the electrode pair at relative ground. The interelectrode conductance is measured in situ using a lock-in amplifier [4]. The alumina layer limits gold deposition to the edges of the electrodes, and mitigates background solution conduction. An analogous layer requiring plasma deposition and a focused ion beam has been used in other junction experiments [5] . Evaporating the dielectric layer yields a similar result with substantially fewer steps. Average resistivity of the electrodeposited gold at the electrode edges at $4.2 \mathrm{~K}$ is approximately $20 \mu \Omega-\mathrm{cm}$.

When an atomic-scale connection is formed initially between the electrodes, discrete conductance steps on the order of $G_{0}$ are observed (Fig. (1). By adjusting the deposition current, we stop the growth near a specific junction 


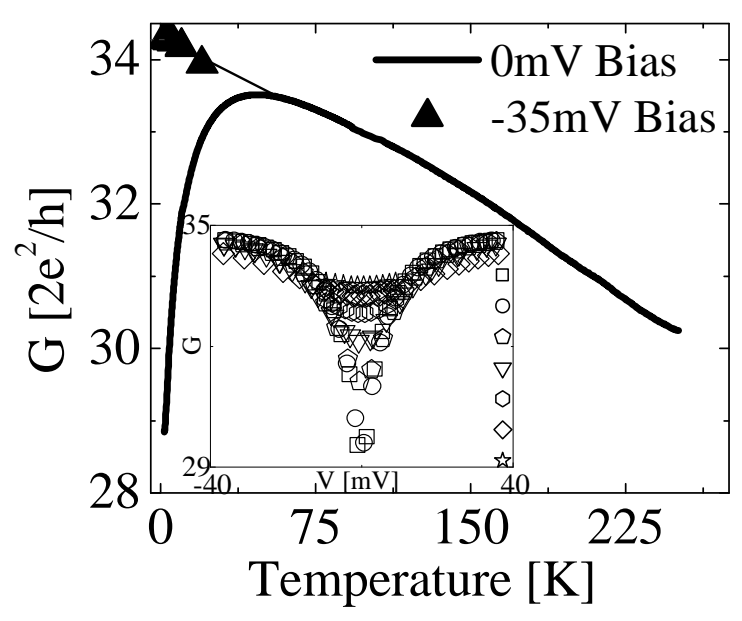

FIG. 2: Temperature sweeps of $d I / d V$ at low and high dc bias in a relatively large junction; Inset: bias sweeps at various $T$ showing zero-bias anomaly. Symbols top to bottom are for $T=1.8 \mathrm{~K}, 2.5 \mathrm{~K}$, $6 \mathrm{~K}, 10 \mathrm{~K}, 15 \mathrm{~K}, 20 \mathrm{~K}$, and $25 \mathrm{~K}$.

conductance. The final step is the removal of the solution using dry nitrogen gas.

At room temperature junctions of a few $G_{0}$ are stable as long as tens of minutes, longer than those made in UHV [2, 3, 9], as expected in "dirty" junctions [10]. Discrete spontaneous conductance changes are frequently observed, including breakage and coalescence. Such changes are much more rare at lower temperatures, consistent with junctions made from a small number of gold atoms that can diffuse at room temperature.

We have also fabricated gold nanojunctions by an alternative electrochemical method 7] (labeled "BT"), using a $0.2 \mathrm{M} \mathrm{HCl}$ solution and applying a bias voltage between the source and drain. By varying a series resistor, nanojunctions with various conductance values resulted. The stability of these nanojunctions is comparable to those described above.

We have measured ten surviving nanojunctions with room temperature conductances ranging from 0.5 to $200 G_{0}$. Thermal contraction effects upon cooling appear to be negligible, except as discussed below. We measure differential conductance $G(V, T) \equiv d I / d V$, as a function of temperature and dc bias voltage using a quasi- 4 terminal lock-in method to eliminate the resistance of the cryostat leads. All nanojunctions show Ohmic behavior up to $200 \mathrm{mV}$ at $300 \mathrm{~K}$. Samples with $G(T=300 \mathrm{~K})>$ $100 G_{0}$ exhibit metallic behavior from $300 \mathrm{~K}$ to $1.8 \mathrm{~K}$. In smaller nanojunctions, however, we find a suppression of the zero-bias conductance below $50 \mathrm{~K}$. This suppression, shown in Fig. 2 is logarithmic in $T$. Metallic behavior in $G(V, T)$ is recovered when a $-35 \mathrm{mV}$ dc bias is applied. We estimate the cross-section $A$ of this junction to be $3 \mathrm{~nm}^{2}$ by a Sharvin relation 11, 12] for a ballistic point contact $(w, L<<\ell$, the elastic mean free path), $G / G_{0}=A k_{\mathrm{F}}^{2} / 4 \pi$, and $k_{\mathrm{F}}=1.2 \times 10^{10} \mathrm{~m}^{-1}$. The inset shows $G(V, T)$ for this junction below $30 \mathrm{~K}$. The suppres-

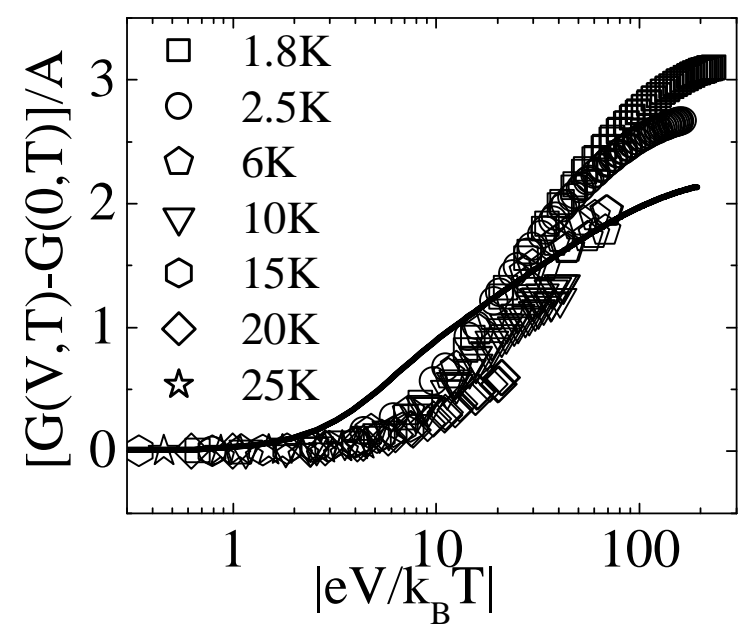

FIG. 3: Scaled ZBA data, showing collapse to one curve at low values of $T, V$, similar to that in Ref. [13]. Solid line shows best fit to the treatment of Ref. 15.

sion becomes more pronounced as $T \rightarrow 0$, reaching about $15 \%$ at $1.8 \mathrm{~K}$. A substantial low temperature ZBA is observed in all samples with $G(300 \mathrm{~K})<\sim 30 G_{0}$ made using the $\mathrm{M}$ electrochemistry. The ZBAs in the BT junctions are much smaller, about $0.2 \%$ at $1.8 \mathrm{~K}$. The application of a constant 8.5 Tesla magnetic field perpendicular to the nanojunction has no detectable effect on the ZBA.

To understand the ZBA we observe in Fig. 2] we consider the analysis in Ref. 13] of electronic transport in larger gold quasi-2d diffusive nanobridges. The ZBAs in such nanobridges are ascribed 13 to perturbative electron-electron interaction corrections to the density of states (DOS) 14 in those devices. At finite temperature Weber et al. predict the conductance of the nanobridge to scale as $[G(V, T)-G(0, T)] / A=f\left(e V / k_{\mathrm{B}} T\right)$, where $A$ describes the temperature dependence of the zero bias conductance, $\left(G(0, T)=G_{*}+A \ln (T)\right)$ and $f(x)$ is a function determined by the diffusive nature of the nanobridge. Figure 3 shows our data scaled this way. At $\left|e V / k_{\mathrm{B}} T\right|<1, G$ is essentially constant, corresponding to thermal smearing. At $\left|e V / k_{\mathrm{B}} T\right|>1, G$ is roughly logarithmic, qualitatively consistent with the data of Weber et al. We do not expect quantitative agreement, since our nanojunction is nondiffusive. A treatment of interactionbased ZBAs in $G>>G_{0}$ coherent systems 15 fits well with $G(0, T)$, but deviates systematically from the data at finite bias, a trend under investigation.

Atomic-scale junctions with correspondingly lower values of $G(300 \mathrm{~K})$ exhibit larger ZBAs at low temperatures. Figure 4 shows $G$ of one informative atomic-scale nanojunction $\left(G(T=300 \mathrm{~K}) \approx 1 G_{0}\right)$ as a function of temperature cycling. During the initial cool down (1), the $G(0, T)$ is nearly $T$-independent until below $50 \mathrm{~K}$, when a substantial drop appears as in previous samples. Upon cooling to $15 \mathrm{~K}, G$ spontaneously increases by about $0.6 G_{0}$. As $T$ is further reduced, $G$ continues to decrease, but from a higher baseline conductance. When 

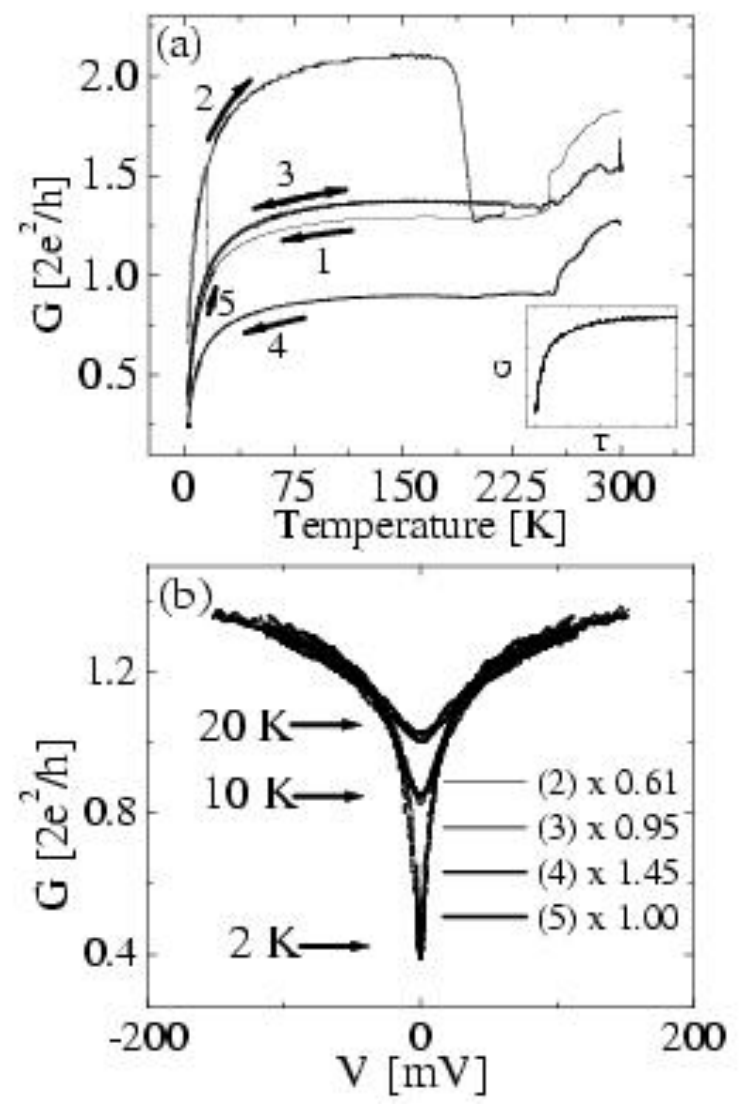

FIG. 4: (a) Temperature cycling of a $G(300 \mathrm{~K}) \approx 1 G_{0}$ junction, showing discrete, hysteretic behavior; inset: all the branches of $G(0, T)$ collapse onto a single curve when each is scaled by a multiplicative constant; (b) ZBA data from the junction configurations of (a), all collapsed by scaling factors obtained from $G(V=0, T)$.

the nanojunction is warmed (2), it appears to have a high temperature $G \approx 2 G_{0}$. However, at $220 \mathrm{~K}$ the conductance of the nanojunction spontaneously decreases by about $0.85 G_{0}$, returning near its original value. Additional cycling and exposure to light produce further branches of $G(V=0, T)(3,4,5)$. Remarkably, all these $G(V=0, T)$ curves collapse onto one curve (see inset) when each branch of $G(T)$ is multiplied by a constant.

The discrete changes confirm the atomic scale of the nanojunction. The addition of a single partially transmitting channel as $T \rightarrow 0$ presumably occurs as thermal contraction brings the source and drain closer together. The loss of the channel on warming supports this view, with thermal expansion now stretching the junction. This sort of hysteresis behavior is seen in break junction measurements.
For each conductance branch described above, the ZBA is measured at several temperatures. Figure 廿p shows $G(V, T)$ at $2 \mathrm{~K}, 10 \mathrm{~K}$, and $20 \mathrm{~K}$ for this nanojunction in its various configurations. Scaling by the factors used to collapse the $G(V=0, T)$ branches, the ZBA data also collapse. Clearly $G(V, T)$ is of the form $B \times g(V, T)$, where $B$ is a constant characteristic of a particular configuration of junction atoms, and $g(V, T)$ is a single function applicable to all the conductance branches. We hypothesize that the size and form of $g(V, T)$ is related to impurities and disorder in the leads, and that such disorder is fabrication method- and sample-dependent. Note that $\Delta G / G$ for this ZBA is nearly $70 \%$; we find similar nonperturbative ( $>60 \%$ ) ZBAs in three other M samples with similar values of $G(300 \mathrm{~K})$. Such large suppressions are never seen in higher conductance junctions.

A natural interpretation of the scaling described above is that these nanojunctions probe the local DOS of the leads. The differential conductance measurements are then analogous to the tunneling conductance experiments used to confirm the perturbative DOS anomalies 14 in planar tunnel junctions. The transmittance of the junction is approximately independent of temperature and energy, and depends on the precise configuration of two or three mobile atoms. The DOS at the junction-lead boundary, however, is temperature and energy dependent, and is determined by disorder "built in" during the electrodeposition process. It is this DOS that develops a nonperturbative suppression at low temperatures. A further discussion of this will be presented elsewhere 16 .

A simple and reliable electrochemical method is used to fabricate atomic size nanojunctions with room temperature conductance ranging from $0.5 G_{0}$ to $200 G_{0}$. The nanojunctions are stable in ambient environment for tens of minutes and indefinitely below $200 \mathrm{~K}$ under vacuum. Differential conductance measurements while varying $T$ and $V$ reveal zero-bias anomalies at low temperatures. Scaling of data from multiple configurations of a particular sample shows that large ZBAs in atomic-scale junctions are due to nonperturbative corrections to the local density of states in the disordered metal leads near the junctions. The disorder in the leads is presumably due to the particular solution-based electrochemistry used. These nontrivial DOS properties imply that electrochemically fabricated nanoelectrodes may be poor choices for use in molecular electronics experiments.

The authors gratefully acknowledge the support of the Robert A. Welch Foundation and the Research Corporation.
[1] J.K. Gimzewski and R. Moller. Phys. Rev. B 36, 1284 (1987).

[2] J.M. van Ruitenbeek, A. Alvarez, I. Piñeyro, C. Grahmann, P. Joyez, M.H. Devoret, D. Esteve, and C. Urbina.
Rev. Sci. Instr. 67, 108 (1996).

[3] For a review, see J.M. van Ruitenbeek, in Metal Clusters on Surfaces: Structure, Quantum Properties, Physical Chemistry, K.H.Meiwes-Broer, ed. (Springer-Verlag, 
Berlin, 2000).

[4] A.F. Morpurgo, C.M. Marcus, and D.B. Robinson. App. Phys. Lett. 74, 2084 (1999).

[5] C.Z. Li, H.X. He, A. Bogozi, J.S. Bunch, and N.J. Tao. App. Phys. Lett. 76, 1333 (2000).

[6] M.W. Wu and L.L. Sohn. IEEE Elect. Dev. Lett. 21, 277 (2000).

[7] S. Boussaad and N. J. Tao. Appl. Phys. Lett. 80, 2398 (2002).

[8] Y.V. Kervennic, H.S.J. Van der Zant, A.F. Morpurgo, L. Gurevich, and L.P. Kouwenhoven. Appl. Phys. Lett. 80, 321 (2002).

[9] C. A. Stafford. Phys. Stat.Sol. B 230, 481 (2002).

[10] K. Hansen, S. K. Nielsen, M. Brandbyge, E. Laegsgaard,
I. Stensgaard, and F. Besenbacher. Appl. Phys. Lett. 77, 708 (2000).

[11] Y.V. Sharvin. Sov. Phys. JETP 21, 655 (1965).

[12] U. Murek, R. Schäfer, and W. Langheinrich. Phys. Rev. Lett. 70, 841 (1993).

[13] H. B. Weber, R. Häussler, H. V. Löhneysen, and J. Kroha. Phys. Rev. B 63, 165426 (2001).

[14] B. L. Altshuler and A. G. Aronov, in Electron-Electron Interactions in Disordered Systems, A. L. Efros and M. Pollak, eds. (North Holland, New York, 1985).

[15] D.S. Golubev and A.D. Zaikin. Phys. Rev. Lett. 86, 4887 (2001).

[16] L.H. Yu and D. Natelson. In preparation. 